\title{
On the Application of Compressed Sensing in Communication Networks
}

\author{
Xiao Wang ${ }^{\dagger \ddagger}$, Zhifeng Zhao ${ }^{\dagger \dagger}$, Ning Zhao ${ }^{\dagger \dagger}$, and Honggang Zhang ${ }^{\dagger *}$ \\ ${ }^{\dagger}$ York-Zhejiang Lab for Cognitive Radio and Green Communications \\ ${ }^{\ddagger}$ Department of Information Science and Electronic Engineering \\ Zhejiang University \\ Zheda Road 38, Hangzhou, 310027 China \\ Correspondence Email: zhaozf@zju.edu.cn
}

\begin{abstract}
Compressed sensing (CS) is an emerging theory based on the fact that the salient information of a signal can be preserved in a relatively small number of linear projections. Compressed sensing has been well used in the area of image compression and signal processing in the past few years. Recently, compressed sensing has been earning ever-increasing interests in the area of wireless communication networks. According to its advantageous characteristics, compressed sensing is able to play significant role in the fields like wireless channel estimation, signal detection, data gathering, network monitoring, and so on. This study describes current researches on the applications of compressed sensing in wireless communication networks, and then enumerates burning questions and the master keys of their corresponding solutions in these fields. Accordingly, we first introduce the basic approach of compressed sensing, and then summarize recent technical advancements of compressed sensing schemes and their applications in wireless communication networks. We assort these techniques according to the general OSI (Interconnection Reference Model) network model. In the end, we analyze problems and potential applications of compressed sensing, including novel methods for efficient data gathering by executing compressed sensing with random routing in wireless sensor networking environment.
\end{abstract}

Keywords-compressed sensing; communication networks; signal detection; channel estimation; data gathering; network monitoring

\section{INTRODUCTION}

In the traditional process of sampling, Nyquist-Shannon theorem has to be obeyed, which means that the sampling rate mush be at least twice the signal bandwidth. Nevertheless, due to the great increasing demand for information, the acquisition, processing and transmission of signal guided by this theorem are faced with serious challenges. Therefore, compressed sensing (CS) has been proposed by Donoho, Candes and Tao in 2004 [1]-[3], as a novel technique to capture and to represent compressible signals at a rate significantly below the Nyquist

This paper is supported by the Key Project of Education Department of Zhejiang Province (No. 20070247), the Key Project of the Office of Science and Technology of Zhejiang Province (No. 2008C01051-3), and the Key Project of the Ministry of Industry and Information Technology of China ("Research on Broadband Wireless Access Systems Architecture and Framework for Supporting Broadcasting Services” No.2009ZX03005-004). rate. CS exploits the inherent scarcity features and the correlation between some input signals to compress such data by matrix, which is formed by non-adaptive linear projections, and then reconstruct these signals through an optimization process. CS theory asserts that certain signals and images can be recovered from far fewer samples or measurements than traditional methods use, when the original data and the compression matrix have certain properties.

In 2004, CS was first proposed for efficient storage and compression of digital images, which show high space correlation. In the following few years, CS has expressed many advantages and its application has prevailed in these fields, with the development of plenteous novel techniques, such as developing simpler, smaller, and cheaper digital cameras, novel analog-to-digital (A/D) converter architectures and so on. Recently, interests in CS as a disruptive approach in wireless communication networks and signal processing begin to grow. Traditionally, data are first compressed and then transmitted to a given destination. CS makes it possible to executed data compression with data transmission jointly. Novel approaches using CS no longer need any correlation information of the input signal and can thereby significantly cut down many transmission overhead costs. In a word, the advantages of CS make it very promising for wireless communication networks, especially for those composed of large numbers of distributed nodes.

This overview paper intends to summarize the state-of-theart researches on CS applications in wireless communication networks. Corresponding to the general OSI (Interconnection Reference Model) network model, we describe in sequence the relevant study of CS applications in physical layer, MAC layer, network layer, as well as application layer, respectively. Then, we showcase problems as well as the corresponding solutions in the applications of CS, especially, a unique idea in efficiently exploiting CS approach with random routing to gathering measuring data in wireless sensor networks (WSN).

The remainder of this paper is structured as follows. In Section II, we make an introduction on the fundamentals of CS theory, and summarize the reconstruction methods of CS. The applications of CS in physical layer are explained in Section III Section IV expresses the problems and potential applications of $\mathrm{CS}$ in wireless communication networks. Conclusion and future works are given in section $\mathrm{V}$. 

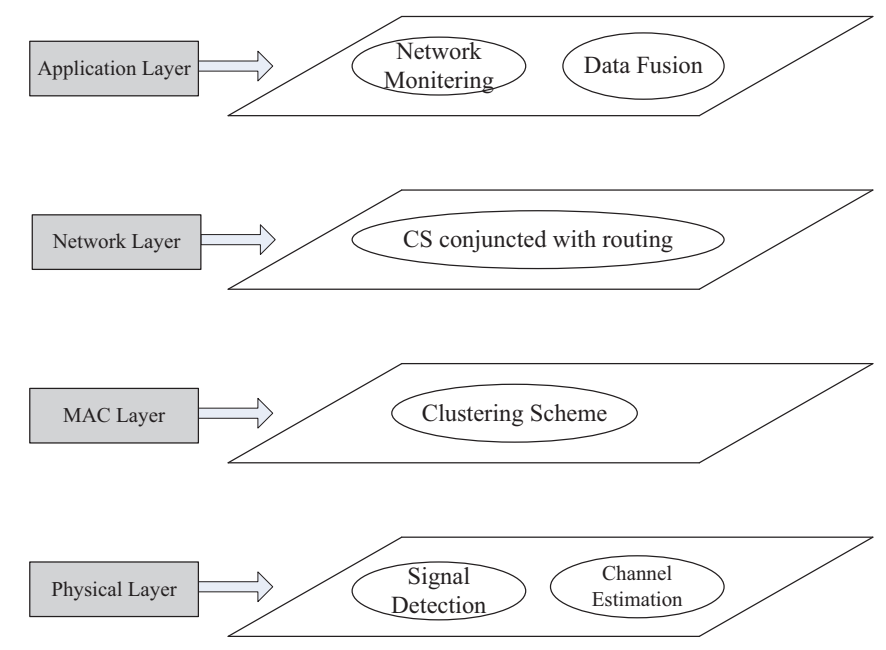

Figure 1. Applications of CS in communication networks according to OSI network model

\section{Compressive Sensing Theory}

The celebrated Nyquist-Shannon theorem underlies most signal acquisition protocols. Nevertheless, the acquisition, processing and transmission of signal guided by this theorem are faced with many critical challenges due to the exponentially increasing demand for all kinds of information. As an emerging technique, CS theory states that one can directly acquire information about certain signals and then recover it from far fewer measurements than traditional methods. This novel theory involves three important aspects as follows:

\section{A. Sparse Representation of the Signal}

To simplify the statement, consider a real-valued discretetime signal $\mathrm{x}$ with a finite-length $\mathrm{N}$, expressed as $\mathrm{x}(\mathrm{n}), \mathrm{n}=1$, $2, \ldots, \mathrm{N}$, which can be viewed as an $N \times 1$ column vector in $\mathfrak{R}^{N}$. Any signal $x \in \mathfrak{R}^{N}$ can be expanded in an orthonormal basis $\Psi=\left[\psi_{1}, \psi_{2}, \ldots, \psi_{N}\right]$ as follows [4]:

$$
x=\sum_{i=1}^{N} \alpha_{i} \psi_{i}=\Psi \alpha
$$

where $\alpha$ is the coefficient sequence of $\mathrm{x}, \alpha_{i}=\left\langle x, \psi_{i}\right\rangle$. When only $\mathrm{K}$ of the ${ }^{\alpha_{i}}$ coefficients are nonzero, $\mathrm{K}<<\mathrm{N}$, the signal $\mathrm{x}$ is compressible and has a sparse representation, called $\mathrm{K}$-sparse. Although most of the natural signals are not sparse in a strict sense, they are always have concise representations when expressed in a proper sparsifying basis $\Psi$, in the sense that the sorted magnitudes of $\alpha_{i}$ decay quickly [5]. Sparscity determines the efficiency of signal acquisition and decreases the needed resource for storage and transmission.

\section{B. Sampling and Sensing Matrix}

Via directly condensing signals into a small amount of data, the useful information in the compressible signals can be captured, which employs non-adaptive linear projections. The sampled values of $\mathrm{x}$ can be expressed as [4]

$$
y=\Phi x=\Phi \Psi \alpha=\Theta \alpha
$$

where $\mathrm{y}$ is an $M \times 1$ column vector and $\Phi$ is an $M \times N$ matrix that is fixed and independent of the signal $x$. Since $\mathrm{M}<<\mathrm{N}$, generally, recovering $\mathrm{x}$ from $\mathrm{y}$ is an ill-posed problem. However, within the framework of CS theory, due to the fact that $\mathrm{x}$ is K-sparse, signal recovery can actually be made possible when the matrix $\Theta=\Phi \Psi$ obeys the rule of restricted isometry property (RIP) or the sensing matrix $\Phi$ is in incoherence with the basis $\Psi$. The smaller the coherence, the fewer samples are needed [5].

\section{Signal Reconstruction}

If the RIP holds or $\Phi$ is incoherent to $\Psi$, the signal can be recovered by $l_{1}-$ norm minimization, namely:

$$
\min _{\alpha}\|\alpha\|_{l_{1} \quad \text { s.t. }} y=\Phi \Psi \alpha
$$

This is a convex optimization problem which can exactly recover the sparse or compressible signal with high probability [1] [3]. Tropp and Gilbert came up with matching pursuit (MP) [6] algorithm and orthogonal matching pursuit (OMP) [7] algorithm that can be fast and easily implemented. Chaining pursuit (CP) [8] algorithm is more efficient for very sparse signal. Recently, different kinds of new or improved compressed signal reconstruction algorithms have sprung up. Readers can refer to the related articles for more detailed information about the different reconstruction algorithms.

\section{Applications of CS in Wireless Communication Networks}

CS is originally proposed to be used in signal and image processing domains where compression is well-performed based on transformation, reflecting the necessary condition under which CS can be effective is that the input data are sparse or highly compressible in certain basis or dictionary. Recently, the advantages of CS have also attracted everincreasing attentions of researchers in the domain of wireless communication networks. In general, communication network is made up of two types of key components: node and communication links, as illustrated in Fig. 2. By analogy, to employ CS in wireless communication networks, compressing bases for the networked data should be completed for achieving sparsity quality. The idea of associating a graph with a given network can make great contribution to the design of transformation bases of networked data. The nodes in the network can be represented by the vertices of the graph, and the relationships between the data at adjacent nodes can be represented by the edges of the graph. As a result, effective sparsifying transformations for the networked data are 
available, so that CS is applicable to wireless communication networks [9].

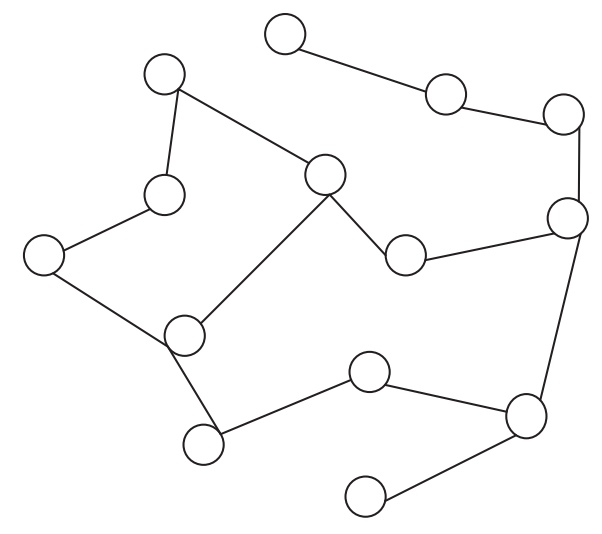

Figure 2. Communication network

Since CS has been earning more and more interests in the area of wireless communication networks, a plenty of researches focused on how to utilize CS efficiently in this area have been carried out. In regard to the OSI-layered network model, we will summarize the relevant researches on the applications of CS in wireless communication networks in the four layers: physical layer, MAC layer, network layer, and application layer, respectively.

\section{A. CS Used in Physical Layer}

In physical layer, there are many cases where a large amount of information should be processed to obtain the interior characteristics, such as signal detection and channel estimation. These problems share a common feature that the number of the input information is really huge but the information is sparse. Forasmuch, CS can be used in these areas to cut down the signal processing cost as well as to increase efficiency.

\section{1) Signal Detection}

In signal detection problems, the signal that is desired to seek from huge amount of rough data is sparse. Accordingly, CS can solve some signal detection problems under particular conditions.

\section{a)
Signals}

a) The Identification of Frequency Hopping

In particularly, CS can be used in the identification of Frequency Hopping (FH) signals, based on the fact that the FH signals needed to be detected are sparse in the local Fourier basis [10]. The sparsity of FH signals means that the signal at any single hop consists of only one sinusoid, whose bandwidth is extremely smaller than that of the whole signal. Therefore, by choosing local Fourier basis to be $\Psi$, and random Gaussian matrix to be $\Phi$, given the hop interval, the FH signals can be identified and the hopping frequencies can be estimated using compressive measurements without reconstructing the signals. In this way, much more energy is saved.

\section{b) Detection in On-Off Random Access Channels}

Regarding a typical on-off random multiple access channel, where $n$ users communicate simultaneously to a single receiver over $m$ degrees of freedom, sparsity detection algorithms, such as orthogonal matching pursuit being used as tractable multiuser detection schemes, can achieve significantly better performance than single-user detection. In addition, the sequential OMP, illustrating that iterative detection combined with power ordering or power shaping, potentially can improve the SNR performance to a great extent [11].

\section{c) Spectrum Sensing in Cognitive Radio}

In the field of cognitive radio (CR) networks, fast and accurate spectrum sensing over a wide bandwidth is extremely important. Fortunately, the bandwidth of the existing signal is very small, comparing with the bandwidth of the whole channel, which means the signal data needed to detected are compressible. Capitalizing on the sparseness of the signal spectrum, CS technique can be employed in spectrum sensing [12][13]. Furthermore it is anticipated that distributed compressive spectrum sensing scheme will play a key role in the complicated heterogeneous networking scenarios, where each CR sensing node transforms the received analog signal from the licensed primary system into a digital signal, using an analog-to-digital information converter, in collaborative manner [14]. The autocorrelation of the compressed signal is then collected from each $\mathrm{CR}$ node at a fusion center. A compressive sampling recovery algorithm that exploits joint sparsity is then employed to reconstruct an estimation of the signal spectrum and used to make a decision on the spectrum occupancy. This scheme is much better than the former compressive spectrum sensing scheme at a single CR, because it obtains spatial diversity in a cooperative mode. It is also studied that CS can be exploited in spectrum sensing in the view of multi-path fading and others, and is authenticated that CS is capable to win an significant performance in repelling such fading.

\section{d) Sparse Event Detection in WSNs}

To detect an event which is sparse, CS is no doubt a very promising technique because of its ability to cut down the number of measurements and its efficiency without prior knowledge as well as overhead control. In particular scenarios, certain prior information of the input data is available. Taking advantage of these prior information, the reconstruction method of CS can be ameliorated to reduce much more cost and at the same time achieve much higher efficiency. It is proposed that in some event detection problems, the value of the input signal is either 1 or 0 , and the distribution probability of the input signal is available. Taking these prior information into account, Bayesian Compressed sensing (BCS), which is much more efficient than traditional reconstruction algorithms, can be utilized. Also, the scenario in which the potential position of the sparse value of the signal is available in advance is studied. In this case, Weighted Basic Pursuit (WBP) algorithm is designed to boost the efficiency of BP algorithm. In a word, taking the advantage of certain knowledge of the input data, the performance of CS can be heightened to a great extend.

\section{2) Channel Estimation}

In most scenarios in communications networks, the number of channels which are used is relatively small compared with the number of the total channels which is vacant at a particular time and space. Therefore, when dealing with channel 
estimation problems, CS can be exploited, since the occupation information of the channels is compressible. Especially in the realms like ultra-wideband (UWB) communications and cognitive radio networks where the channel band is really wide and dynamic, the advantages of CS techniques have generated a plenty of interests.

Since CS does not require any knowledge of the underlying multipath channel, based on the fact that the physical multipath channels exhibit a sparse structure in angle-delayDoppler spreading, especially at large signal space dimensions, it is advantageous to utilize sparse channel estimation methods based on convex/linear programming, which can be proved to outperform the existing training-based methods in quantitative error bounds [15].

Basically, propagation media such as those in underwater acoustic communications (UWA) and ultra-wideband communications are characterized by sparse channels. UWA channels exhibit large delay spreads and multipath propagation. CS algorithms in form of Orthogonal Matching Pursuit and Basis Pursuit (BP), can handle such channels with significant Doppler spread, when coupled with a channel equalizer mitigating inter-carrier interference, as demonstrated in [16] using numerical simulation and experiments. Although CS still has flaws in its present form and the necessary adaptation to sparse channel as an estimator for highly sparse channels, in higher SNR region, its performance is validated to be able to achieve the Cramer-Rao bound for the structured estimation of channel response with considerable computational efficiency. Moreover, [17] made great contribution to exploiting the channel itself as part of compressed sensing in UWB, through waveform-based pre-coding at the transmitter, resulting in a very simple receiver design.

\section{B. CS Used in MAC Layer}

In MAC layer, $\mathrm{CS}$ can be used in WSNs to reduce measurement cost by minimizing the number of measurements. The studies are focused on addressing the problem of high costs caused by dense measurement when CS is utilized. By dense measurement, each measurement in CS is a linear combination of many (or all) samples of the signal to be reconstructed, which may result in significant transport costs, even though the number of measurements has been minimized. So, when CS is used in multi-hop sensor networks, there is a serious problem. In this case, sparse measurement is needed.

[18] provided an efficient approach based on clustering in WSN to solve the problem of dense measurement. The whole network is divided into clusters of adjacent nodes and forces projections to be obtained only from nodes within a cluster. Two clustering approaches were investigated in [18], square clustering, in which the network is partitioned into certain number of equal-size square regions, and SPT (shortest path tree)-based clustering, in which cluster is formed according to the shortest path tree. Using the distribution of energy of the basis functions with respect to the clusters as a metric, [18] proved that joint reconstruction shows better performance than independent reconstruction, and its method also achieves power savings with localized data aggregation. Most importantly, a method to quantify the level of "energy overlap" between the data gathering clusters and the elementary basis on which the signals are projected was presented, which allows designing efficient clusters once the bases for the signal are known.

\section{CS Used in Network Layer}

In network layer, CS is very promising for jointly acquiring and aggregating data from distributed data sources in a multihop wireless sensor network. Current WSNs comprise hundreds of thousands of independent sensing components which are all capable of generating and communicating data, as in Fig. 3(a), and the number of the sensing components is keep growing. Thereby, research challenges in WSNs revolve around large-scale distributed sources of data and their storage, transmission and retrieval. Researchers are struggling to address the problem of efficiently transmitting or sharing information from and among a vast number of distributed nodes. Based on the fact that the number of nodes in WSNs is very huge, while the information generated by the nodes is almost the same, which indicates that the information of the whole network is compressible, CS shows its great potential to resolve the challenges. CS has the following two advantages in view of the current situation of WSNs. First, data compression and transmission can be executed jointly via CS, so none of the correlation properties of the input signal over the entire network is need. Secondly, CS makes the reconstruction of all sensor readings of the network possible, using much fewer transmissions than traditional routing or aggregation schemes, thereby increasing the efficiency of data gathering solutions.

Based on the theory testified in [19] that sparse random projections are capable for the remarkable results of CS, the exploitation of CS in multi-hop WSNs takes into account network topology and routing, which are used to transport random projections of the sensed data to the sink, as shown by Fig. 3(b), and needs to provide effective method to address the problem of dense measurement, in order to reduce costs and increase efficiency.

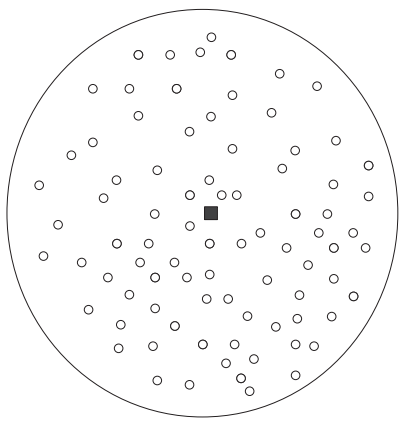

(a)

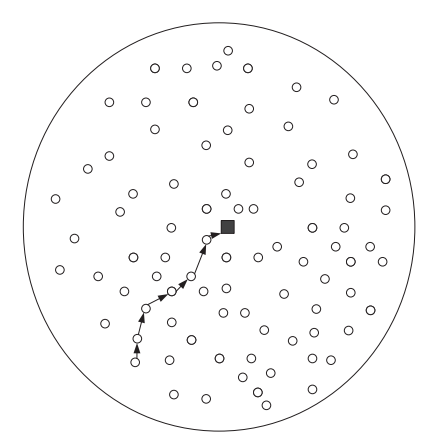

(b)
Figure 3. (a) common structure of WSN. (b) routing in WSN

Using CS in conjunction with routing can be realized through the following scheme. In WSN, each node becomes a source with an appropriate fixed probability. In this case, a fixed number of nodes are chosen to be source nodes, each of which transmits a packet containing the reading of its own 
sensor along its path towards the sink. During the transmission, the value contained in the packet is combined with that of any other node it encounters along the path, at random combination coefficients which are generated at each node respectively. According to CS formalism, these combination coefficients along a path form the corresponding row of the measurement matrix, by setting the elements corresponding to the nodes along the path to be these coefficients and the others to zero. Using CS reconstruction at the sink, the whole information of the network can be reconstructed with good accuracy.

Hence, the measurement matrix highly depends on the network topology and on the selected routing rules. Accordingly, it makes a sense to study a method to combine CS with routing design for energy efficient data gathering in various sensor networking scenarios. However, the effectiveness of standard CS technique suffers limitation when routing costs are considered comparing to random downsampling technique [20][21].

The reason why CS cannot perform well as what has been expected here is that when CS is executed with routing, we can no longer find an appropriate routing scheme to generate the measurement matrix which is incoherent with data basis. Therefore, to meet the conditions under which CS performs well, researches have been conducted in this field with the aim of solving the problem of sparsifying the real data while designing novel routing matrix which is incoherent with the sparsifying basis.

On one hand, several techniques are proposed to sparsify the real data. [22] presented a successful method to sparsify abnormal sensor reading by decomposing them into two vectors: one contains the normal readings which are sparse in a certain transform domain, and the other contains the deviated values of abnormal readings which are sparse in the time domain. In this case, real data sparsity in practical data acquisition is verified, so are the efficiency and robustness of the sensor data reconstruction with and without abnormal readings.

In [23] an approach to signal acquisition by exploiting the spacial attributes of the operational environment called MapBased Compressive Sensing is presented to achieve higher sparsity of signal in representation basis, while finding the optimal mapping is left an endeavor for future work.

On the other hand, it is necessary to investigate novel routing strategies that i)provide a sufficient spatial coverage to enable good coherence properties for compressed sensing along those routes, and ii) have transport costs that do not deviate significantly from those of efficient routing techniques. Moreover, it is reasonable to take advantage of a centralized greedy algorithm for obtaining low coherence projections while simultaneously reducing reconstruction error and communication cost [20]. The centralized algorithm is to iteratively build paths by making a greedy choice for the next node which minimizes the intermediate coherence of the partial measurement matrix with the basis on which data are sparse.

[21] also addressed the joint routing and compression problem by exploiting the spatial correlation among the sensor reading in the $2 \mathrm{D} \mathrm{WSN}$. In detail, real signal analysis is addressed, and furthermore, the conditions under which CS performs well and under which it fails to improve the performance were also studied.

The study of utilizing CS in conjunction with routing in WSNs has just started. Up to now, CS has not shown its inherent superiority, for no appropriate routing scheme has been designed to ensure the required condition of CS.

\section{CS Used in Application Layer}

In application layer, CS can be used in some scenarios where a number of information, which owns certain features, has to be dealt with. In the relevant researches, CS is starting to be utilized in network monitoring and data fusion.

\section{1) Network Monitoring}

Network monitoring is to observe and obtain network characteristics. It is not practical to directly monitor a network, at either path level or link level. In respect of the idea that the messages of the whole network are compressible, because each link typically is used by many paths, CS is likely to provide an efficient method to infer network characteristics from incomplete or indirect measurements.

Specifically, it is valuable to apply CS to network monitoring [24], addressing the problem of efficient end-to-end network monitoring of the path metrics in large-scale wireless communication networks. By exploiting the notion that the performance on two overlapping paths can be correlated, diffusion wavelet [25] is used to approximate the vector of path metrics using only a few of non-zero coefficients. A diffusion wavelet basis, which is adapt to the monitoring problem exploiting spatial and temporal correlations in the measurement phenomena, was developed to provide a compressible representation of the path metrics. Nonlinear estimation technique using $l_{1}$ minimization is exploited for reconstruction of the whole network including the unmeasured paths. Furthermore, a heuristic path selection algorithm for determining the optimal monitoring strategy is described. As a result, the number of measurements or monitors required to maintain acceptable estimation accuracy is minimized to improve the efficiency of end-to-end delay estimation in IP networks meanwhile reduce the number of hardware monitors required to track bit-error-rates in all-optical networks [11]. All of the results are demonstrated using data from the Abilene backbone network. It is proved that CS can bring in much more accurate estimation with few measurements on real data sets, comparing with the previous network kriging estimator.

\section{2) Data Fusion}

In the current large-scale network, where the number of the components is ever-growing, countless messages should be transmitted to the data fusion center, and a mass of control massages are needed. How to efficiently compress the data is the most serious problem. CS offers an efficient approach to dealing with the problem in storage, transmission and retrieval of the huge amount of data, for the networked data are compressible. It is expected that the networked data can be expresses by a few of big coefficients in certain basis, such as spatial compression [26], graph wavelets [27], and diffusion wavelets [25]. For example, when the network topology is a 
regular lattice, the data is compressive by using the tools like Discrete Fourier Transform (DFT) or Discrete Wavelet Transform (DWT). More generally, graph wavelets can adapt the design principles of the DWT to arbitrary graphs. Diffusion wavelets also provide an alternative approach to constructing a multi-scale representation for the networked data defined on a graph.

CS shows great advantages in data fusion. Different from the other decentralized compression strategies, such as SlepianWolf coding, which need a prior knowledge of the correlations between data at different nodes, CS needs no priori knowledge, which has been of increasing interests recently [28]-[32], and CS offers two highly desirable advantages for networked data analysis: one is decentralized, meaning that distributed data can be encoded without a central controller; the other is universal, in the sense that sampling does not require a priori knowledge or assumptions about the data.

Several approaches [9] in the action of networked data compression are presented in the recent researches.

First, in any general multi-hop networks, each of the sensors locally generates its information by multiplying its data with the corresponding element of the compressing matrix. Then the local information is simultaneously aggregated and distributed across the network by randomized gossip algorithm [33]. When randomized gossip terminates, each node in the network knows all of the data in the network, and then $\mathrm{M}$ of the nodes are randomly chosen to transmit their information to the sink.

Secondly, a large number of nodes are sending data to the fusion center. In this case, analog fashion is used [9], which corresponds to a completely decentralized way of delivering $\mathrm{M}$ random projections of the sensed data to the fusion center by employing $M$ (network) transmissions. In other words, this approach means that all of the $\mathrm{N}$ nodes transmit their sensing data once per time-slot during $\mathrm{k}$ time-slots.

Thirdly, in [22] compressive data gathering schemes over chain topology as well as grid topology were proposed to reduce global scale communication cost without introducing intensive computation or complicated transmission control. The capacity for WSN was also analyzed, and a capacity gain of $\mathrm{N} / \mathrm{M}$ over baseline transmission can be achieved.

\section{Problems and Potential Applications of CS in Wireless Communication Networks}

Based on its advantages, CS is a very promising technique in wireless communication networks, which has already been vwrufued by a plenty of researches. However, the studies on the applications of CS are just in fewer areas. Even in these areas, a lot of problems are still not been fully settled, limiting the performance of CS. A great number of technical challenges are left for further study.

In physical layer, in signal detection and channel estimation, in case the number of channels is not large enough, the requirement of sparsity cannot be guaranteed, which hurts the advantages of CS.

In MAC layer, the technique of clustering has been proposed to solve the problem of dense measurement. However, how to design efficient clusters is still an open problem, since the efficiency of reconstruction of CS will be seriously influenced by the choosing of basis functions and clustering structure.

In network layer, network topology and routing are considered in various application scenarios of CS, whereas routing strategies which can be well adaptive to CS have not been successfully designed yet. Researches to find such efficient routing schemes are very promising. The deficiency of the state-of-the-art techniques may lie in that the routing schemes are not adaptive to the execution of CS. In detail, existing routing schemes, e.g., the greedy routing algorithm and the shortest path routing algorithm have the problem that, on one hand, the number of sensors contained in each route is not sufficient for the reconstruction of CS data, and on the other hand, the selecting methods of sensors result in that the values of the elements in the measurement matrix are not randomly chosen enough to satisfy the requirement of incoherence in CS theory. To solve this problem, we design random routing schemes executed with CS for data gathering. In these schemes, it is preferred that the nodes included by the selected routs are uniformly distributed, which means that the distribution of the corresponding none-zero values in the measurement matrix are uniform, benefiting the reconstruction performance of CS.

In application layer, data fusion techniques exploiting CS can minimize the number of measurements; however, the fusion center faces a huge challenge in the reconstruction of the compressed message, which requires a lot of computing. In this case, the fusion center should possess the ability to handle large amounts of calculations efficiently and immediately. There is also another serious problem that has not been paid enough attention: neither missing nor error in data transmission is considered in existing data fusion techniques while exploiting CS.

In one word, to efficiently utilize CS in any field, the basic conditions under which CS can perform well should be met. Specifically, the input data must be sparse in particular basis, and at the same time, the sparsifying basis must be incoherent with the measurement matrix. Further researches on the application of CS in wireless communication networks should also be based on finding effective methods to meet the two basic requirements of $\mathrm{CS}$.

\section{CONCLUSION AND FUTURE WORK}

In this work, we studied the techniques of using CS in communication networks. We summarized the researches in the four layers according to the OSI network model, respectively. In the physical layer, $\mathrm{CS}$ has been used in data detection and channel estimation, especially in UWB, UWA and CR. Cluster-based technique using CS is studied in the MAC layer. In the network layer, researches are centralized on data gathering, in order to achieve high efficiency as well as reduce costs. CS is utilized in data fusion and network monitoring in the application layer. In our opinion, the techniques, which utilize CS with routing in data gathering in WSN, is a very promising development. However, the problem of how to combine CS with routing schemes efficiently, i.e. how to choose a proper routing scheme to meet the basic principles of $\mathrm{CS}$, has not been addressed till now to our 
knowledge. We are now studying on a random routing scheme based on grid network as well as a random circle routing technique to fix the problem mentioned above.

\section{References}

[1] D. Donoho, "Compressed sensing," IEEE Trans. on Information Theory, vol. 52, no. 4, pp. 4036-4048, 2006.

[2] E. Cand'es and T. Tao, "Near optimal signal recovery from random projections: Universal encoding strategies?" IEEE Trans. On Information Theory, vol. 52, no. 12, pp. 5406-5425, 2006.

[3] E. Cand'es, J. Romberg, and T. Tao, "Robust uncertainty principles: Exact signal reconstruction from highly incomplete frequency information," IEEE Trans. on Information Theory, vol. 52, no. 2, pp. 489-509, 2006.

[4] R. Baraniuk. "A lecture on compressive sensing," IEEE Signal Processing Magazine, 24(4):118-121. 2007.

[5] E. J. Candes, M. B. Wakin. "An introduction to compressive sampling," IEEE Signal Processing Magazine, March, 2008:21-23.

[6] R. Nef, A. Zakhor. "Very low bit rate video coding based on matching pursuits," IEEE Transactions on Circuits and Systems for Video Technology, 1997, 7(1): 158-171.

[7] J. A. Tropp and A. C. Gilbert, "Signal recovery from partial information by orthogonal matching pursuit," IEEE Trans. Inform. Theory, vol 53, pp. 4655-4666, 2007.

[8] C. Gilbert, M. J. Slrauss, J A Tropp, etc., "Algorithmic linear dimension reduction in the norm for sparse vectors," 44th Annual Allerton Conference on Communication, Control, and Computing, 2006.

[9] J. Haupt, W. Bajwa, M. Rabbat, and R. Nowak, "Compressive Sensing for Networked Data: a Different Approach to Decentralized Compression," IEEE Signal Processing Magazine, vol. 25, no. 2, pp. 92-101, 2008.

[10] J.Yuan, P.Tian, and H.Yu, "The identification of frequency hopping signal using compressive sensing," Communications and Network,2009,52-56

[11] A. K. Fletcher, S. Rangan, and V. K. Goyal, "On-off random access channels: A compressed sensing framework," arXiv:0903.1022v1 [cs.IT]., Mar. 2009.

[12] Z. Tian and G. B. Giannakis, "Compressed sensing for wideband cognitive radios," Proceedings of IEEE ICASSP, 2007.

[13] Y. L. Polo, Y. Wang, A. Pandharipande and G. Leus, "Compressive Wide-Band Spectrum Sensing," International Conference on Acoustics, Speech, and Signal Processing, 2009.

[14] Y. Wang, A. Pandharipande, Y. L. Polo, and G. Leus, "Distributed compressive wide-band spectrum sensing," Proc. Information Theory and Applications Workshop, 2009, 178-183

[15] W. U. Bajwa, A. M. Sayeed, and R. Nowak, "Compressed sensing of wireless channels in time, frequency, and space," Proc. 42nd Asilomar Conf. Signals, Systems, and Computers, Oct. 2008.

[16] C. R. Berger, S. Zhou, J. C. Preisig, and P. Willett, "Sparse channel estimation for multicarrier underwater acoustic communication: From subspace methods to compressed sensing," Proc. of MTS/IEEE OCEANS conference, Bremen, Germany, May 11-14, 2009.
[17] P. Zhang, P. Hu, R. C. Qiu, and B. M. Sadler, "A compressed sensing based ultra-wideband communication system," ICC '08. Dresden, Germany, Jun. 2009.

[18] S Lee, S Pattem, and M Sathiamoorthy, "Spatially-localized compressed sensing and routing in multi-hop sensor networks," 3rd International Conference on Geosensor Networks (GSN), 2009.

[19] W. Wang, M. Garofalakis, and K. Ramchandran, "Distributed sparse random projections for refinable approximation," Proc. IPSN'07, Cambridge, MA, April 2007, pp. 331-339.

[20] S. Lee, S. Pattem, M. Sathiamoorthy, B. Krishnamachari and A. Ortega, "Compressed sensing and routing in multi-hop networks," Technical Report, University of Southern California, 2009.

[21] G. Quer, R. Masiero, D. Munaretto, M. Rossi, J. Widmer, and M. Zorzi, "On the Interplay Between Routing and Signal Representation for Compressive Sensing in Wireless Sensor Networks," Information Theoryand Applications Workshop (ITA 2009), San Diego, CA, US, Feb. 2009.

[22] C. Luo, F. Wu, J. Sun, and C. Chen. "Compressive data gathering for large-scale wireless sensor networks," MobilCom '09, Sep 2009.

[23] M. Mahmudimanesh, A. Khelil, and N.Yazdani, "Map-Based Compressive Sensing Model for wireless Sensor Network Architecture, A Starting Point," Mobilware 2009 Workshops, Berlin, Germany, April 2009, Revised Selected Papers.

[24] M. Coates, Y. Pointurier, and M. Rabbat, "Compressed Network Monitoring for IP and All-optical Networks," IMC, 2007.

[25] R. Coifman and M. Maggioni, "Diffusion wavelets," Applied Computational and Harmonic Analysis, vol. 21, no. 1, pp. 53-94, July 2006.

[26] R. Wagner, R. Baraniuk, S. Du, D. Johnson, and A. Cohen, "An architecture for distributed wavelet analysis and processing in sensor networks," Proc. IPSN'06, Nashville, TN, April 2006, pp. 243-250.

[27] M. Crovella and E. Kolaczyk, "Graph wavelets for spatial traffic analysis," Proc. IEEE Infocom, vol. 3, Mar. 2003, pp. 1848-1857.

[28] S. S. Pradhan, J. Kusuma, and K. Ramchandran, "Distributed compression in a dense microsensor network," IEEE Signal Processing Mag., vol. 19, no. 2, pp. 51-60, Mar. 2002.

[29] W. U. Bajwa, J. Haupt, A. M. Sayeed, and R. Nowak, "Compressive wireless sensing,” Proc. IPSN'06, Nashville, TN, Apr. 2006, pp. 134142 .

[30] M. Rabbat, J. Haupt, A. Singh, and R. Nowak, "Decentralized compression and predistribution via randomized gossiping," Proc. IPSN'06, Nashville, TN, Apr. 2006, pp. 51-59.

[31] W. U. Bajwa, J. Haupt, A. M. Sayeed, and R. Nowak, "A universal matched source-channel communication scheme for wireless sensor ensembles," in Proc. ICASSP'06, Toulouse, France, May 2006, pp. 1153-1156.

[32] M. F. Duarte, S. Sarvotham, D. Baron, M. B. Wakin, and R. G. Baraniuk, "Distributed Compressed Sensing of Jointly Sparse Signals," Proc. Conf Signals, Systems and Computers Record of the Thirty-Ninth Asilomar Conf, 2005, 1537-1541

[33] S. Boyd, A. Ghosh, B. Prabhakar, and D. Shah, "Randomized gossip algorithms," IEEE Trans. Inform. Theory, vol. 52, no. 6, pp. 2508-2530, June 2006. 